\title{
CP violation in B decays to charmonia at $\mathrm{LHCb}$
}

\author{
Francesca Dordei ${ }^{* \dagger}$ \\ INFN Sezione di Cagliari, Monserrato, Italy and European Organization for Nuclear Research \\ (CERN), Geneva, Switzerland \\ E-mail: francesca.dordei@cern.ch
}

\begin{abstract}
Measurements of $C P$ violation in decays of beauty-hadrons to charmonia final states provide a fundamental test of Standard Model (SM) predictions and represent a sensitive probe to search for physics effects beyond the SM. In these proceedings, precision measurements are presented of several observables related to the so-called unitary triangles, that arise from the unitarity requirements on the $3 \times 3$ Cabibbo-Kobayashi-Maskawa matrix describing the quark mixing. The discussed analyses are based on proton-proton collision data collected during 2011 and 2012 (LHC Run 1) by the LHCb experiment.
\end{abstract}

The 39th International Conference on High Energy Physics (ICHEP2018)

4-11 July, 2018

Seoul, Korea

\footnotetext{
* Speaker.

${ }^{\dagger}$ On behalf of the LHCb collaboration
} 


\section{Introduction}

Although the Standard Model (SM) has been very successful in describing existing data [1], contributions from physics Beyond the Standard Model (BSM) are needed [2] in order to explain, for example, the large matter-antimatter asymmetry that resulted in the matter dominated universe we observe today [3]. In the SM, the only source of $C P$ violation is a complex phase in the CabbiboKobayashi-Maskawa (CKM) quark mixing matrix [5], which is many orders of magnitude too small to match the observed matter-antimatter asymmetry [4]. Most of the BSM physics models available today predict the existence of additional unobserved heavy particles that can appear in internal lines of higher order diagrams contributing to a process (so called box and penguin diagrams), producing sizeable modifications of observables related to these processes, such as $C P$ violating parameters. Therefore, precision measurements of $C P$ violation are mandatory in order to confirm SM predictions and to check whether non-SM contributions are also present. In the following, a selection of recent $\mathrm{LHCb}$ [7] measurements of $C P$ violation in $B$ decays to charmonia, based on 2011 and 2012 proton-proton collisions data, are presented.

\section{CKM metrology}

The unitarity of the CKM matrix gives rise to six orthogonality relations, which can be represented as triangles in the complex plane. The overconstrained determination of the sides and angles of these Unitarity Triangles provides a powerful consistency test of the SM picture of $C P$ violation.

\subsection{Measurement of the CKM phase $\beta$}

The angle $\beta$ is related to CKM matrix elements through the relation $\beta \equiv \arg \left[-\left(V_{c d} V_{c b^{*}}\right) /\left(V_{t d} V_{t b}^{*}\right)\right]$ and it is sensitive to BSM effects in $C P$ violation in mixing and decay. Applying CKM unitarity and using measurements of other CKM-related quantities leads to a SM prediction of $\sin 2 \beta=$ $0.740_{-0.025}^{+0.020}$ [17]. The golden channel to determine this angle is $B^{0} \rightarrow J / \psi K_{s}^{0}$. At LHCb $J / \psi \rightarrow$ $\mu \mu$ decays are selected efficiently and the relatively high branching fraction of decays such as $B^{0} \rightarrow J / \psi K_{s}^{0}$ gives large samples of $b \rightarrow c \bar{c} s$ transitions to be analysed. By measuring the flavourtagged decay-time-dependent $C P$ asymmetry using the full Run 1 dataset, the LHCb collaboration measures [9] the following $\mathrm{CP}$-violating parameters

$C=-0.038 \pm 0.032$ (stat.) \pm 0.005 (syst.) and $S \equiv \sin (2 \beta)=0.731 \pm 0.035$ (stat.) \pm 0.020 (syst.).

The largest systematic uncertainty on the measurement of $\sin (2 \beta)$ arises from possible flavour asymmetries in the background candidate. The LHCb collaboration has also recently measured $S$ and $C$ using $B^{0} \rightarrow J / \psi\left(e^{+} e^{-}\right) K_{s}^{0}$ and $B^{0} \rightarrow \psi(2 S)\left(\mu^{+} \mu^{-}\right) K_{s}^{0}$ decays [10]. The combined $B^{0} \rightarrow$ $[c \bar{c}] K_{S}^{0} C P$-violation observables are determined to be

$$
C\left(B^{0} \rightarrow[c \bar{c}] K_{S}^{0}\right)=-0.017 \pm 0.029 \text { and } S\left(B^{0} \rightarrow[c \bar{c}] K_{S}^{0}\right) \equiv \sin (2 \beta)=0.760 \pm 0.034 .
$$

The individual LHCb measurements and their combination are shown in Figure 1 (left). After Upgrade II, that aims to achieve a total integrated luminosity of $300 \mathrm{fb}^{-1}, \mathrm{LHCb}$ will be able to reach a statistical precision of below 0.003 [19]. The majority of systematic uncertainties depend on the size of control samples, and are therefore not expected to be limiting. 

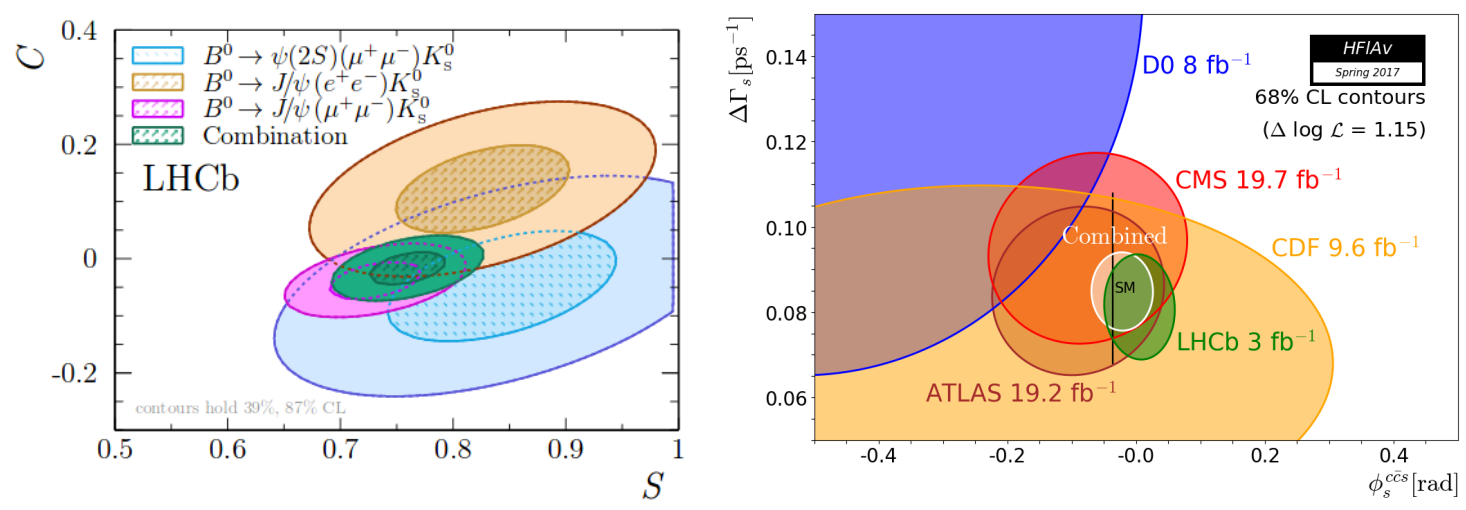

Figure 1: Left: Two-dimensional likelihood scans for the combination of all $B^{0} \rightarrow[c \bar{c}] K_{s}^{0}$ modes analysed by LHCb. Right: Two-dimensional likelihood scans of $\phi_{s}$ and $\Delta \Gamma_{s}$. The white contour represents the HFLAV combination to be compared to the SM prediction (black line).

\subsection{Measurement of the CKM phase $\beta_{s}$}

The corresponding $C P$ observable in the $B_{s}^{0}$ system is called $\beta_{s}=-2 \phi_{s}$ and it is related to CKM matrix elements through the relation $\phi_{s}^{S M} \equiv-2 \arg \left(-\frac{V_{t s} V_{t b^{*}}}{V_{c s} V_{c b}^{*}}\right)$. This angle has been extensively studied using the golden channel $B_{s}^{0} \rightarrow J / \psi \phi$. This decay is a pseudo-scalar to vector-vector decay, so angular momentum conservation implies that the final state is an admixture of $C P$-even and $C P$-odd components. By performing a tagged time-dependent angular analysis, it is possible to statistically disentangle the different $C P$ eigenstates by the differential decay rate for $B_{s}^{0}$ and $\bar{B}_{s}^{0}$ mesons produced as flavour eigenstates at $t=0$. The LHCb collaboration exploited also other decay modes to fully investigate the potentials of Run 1 data. Different measurements have been performed using various $B_{s}^{0}$ finale states like $J / \psi \pi^{+} \pi^{-}$[18], $\psi(2 S) \phi$ [13], $D_{s}^{+} D_{s}^{-}$[14] and $J / \psi K^{+} K^{-}$[15] for the $K^{+} K^{-}$invariant mass region above $1.05 \mathrm{GeV}$. The HFLAV average including all the above mentioned results is $\phi_{s}=-21 \pm 31 \mathrm{mrad}$ [16]. The individual measurements and their HFLAV combination are shown in Figure 1 (right). Despite the impressive progress since the initial measurements performed by CDF and D0, the uncertainty is still much larger than the indirect determination from global fits: $\phi_{S}^{\mathrm{SM}}=-36.5_{-1.2}^{+1.3} \mathrm{mrad}$ [17]. The expected precision on $\phi_{s}$ after Upgrade II will be $\sim 4 \mathrm{mrad}$ from $B_{s}^{0} \rightarrow J / \psi \phi$ alone and $\sim 3 \mathrm{mrad}$ from all modes combined [19]. This will be at the same level as the current precision on the indirect determination based on the CKM fit using tree-level measurements (this in turn is expected to improve with better measurements of other CKM matrix parameters).

\section{Conclusions}

Recent LHCb results of $C P$ violation in the beauty sector have been presented. All measurements are in good agreement with SM predictions. The selection of $C P$ violation measurements presented here is not a complete list of the analysis performed by the LHCb collaboration. Updated results based on the Run 2 data, corresponding to an integrated luminosity of about $6 \mathrm{fb}^{-1}$, are expected soon and will enable even stronger constraints to be made on potential BSM physics contributions. 


\section{References}

[1] M. Tanabashi et al. [Particle Data Group], “The Review of Particle Physics”, Phys. Rev. D 98, 030001 (2018).

[2] A. D. Sakharov, "Violation of $C P$ invariance, $C$ asymmetry, and baryon asymmetry of the universe", Soviet Physics Uspekhi 34, 392 (1991).

[3] A. Riotto and M. Trodden, "Recent progress in baryogenesis", Ann. Rev. Nucl. Part. Sci. 49, 35-75 (1999).

[4] M. P. Gavela, P. Hernandez, J. Orloff and O. Pene, "Standard Model CP violation and baryon asymmetry", Mod. Phys. Lett. A9, 795 (1994).

[5] M. Kobayashi and T. Maskawa, " $C P$ violation in the renormalizable theory of weak interaction", Prog. Theor. Phys. 49, 652 (1973).

[6] U. Nierste, “Three Lectures on Meson Mixing and CKM phenomenology”, arXiv:0904.1869 [hep-ph] (2009).

[7] A. Alves Jr. et al. [LHCb Collaboration], “The LHCb detector at the LHC”, JINST 3, S08005 (2008).

[8] J. Charles et al., "Current status of the Standard Model CKM fit and constraints on $\Delta F=2$ New Physics”, Phys. Rev. D 91, 073007 (2015).

[9] R. Aaij et al. [LHCb Collaboration], "Measurement of $C P$ violation in $B^{0} \rightarrow J / \psi K_{S}^{0}$ decays", Phys. Rev. Lett. 115, 031601 (2015).

[10] R. Aaij et al. [LHCb Collaboration], "Measurement of $C P$ violation in $B^{0} \rightarrow J / \psi\left(e^{+} e^{-}\right) K_{S}^{0}$ and $B^{0} \rightarrow \psi(2 S)\left(\mu^{+} \mu^{-}\right) K_{S}^{0}$ decays", JHEP 11, 170 (2017).

[11] G. Aad et al. [ATLAS Collaboration], "Measurement of the CP-violating phase $\phi_{s}$ and the $B_{s}^{0}$ meson decay width difference with $B_{s}^{0} \rightarrow J / \psi \phi$ decays in ATLAS", JHEP 1608, 147 (2016).

[12] R. Aaij et al. [LHCb Collaboration], "Precision measurement of $C P$ violation in $B_{s}^{0} \rightarrow J / \psi K^{+} K^{-}$ decays", Phys. Rev. Lett. 114, 041801 (2015).

[13] R. Aaij et al. [LHCb Collaboration], "First study of the CP -violating phase and decay-width difference in $B_{s}^{0} \rightarrow \psi(2 S) \phi$ decays", Phys. Lett. B 762, 253 (2016).

[14] R. Aaij et al. [LHCb Collaboration], "Measurement of the $C P$-violating phase $\phi_{s}$ in $\bar{B}_{s}^{0} \rightarrow D_{s}^{+} D_{s}^{-}$ decays", Phys. Rev. Lett. 113, 211801 (2014).

[15] R. Aaij et al. [LHCb Collaboration], "Resonances and $C P$ violation in $B_{s}^{0}$ and $\bar{B}_{s}^{0} \rightarrow J / \psi K^{+} K^{-}$ decays in the mass region above the $\phi(1020)$ ", JHEP 1708, 037 (2017).

[16] Y. Amhis et al., "Averages of $b$-hadron, $c$-hadron, and $\tau$-lepton properties as of summer 2016", arXiv:1612.07233 [hep-ex].

[17] J. Charles et al., "Current status of the Standard Model CKM fit and constraints on $\Delta F=2$ New Physics”, Phys. Rev. D 91, 073007 (2015).

[18] R. Aaij et al. [LHCb Collaboration], "Measurement of the CP-violating phase $\phi_{s}$ in $\bar{B}_{s}^{0} \rightarrow J / \psi \pi^{+} \pi^{-}$ decays", Phys. Lett. B 736, 186 (2014).

[19] R. Aaij et al. [LHCb Collaboration], "Physics case for an LHCb Upgrade II - Opportunities in flavour physics, and beyond, in the HL-LHC era", arXiv:1808.08865 [hep-ex]. 